\title{
State Feedback Stabilization of Switched Systems with Delay: Trajectory Based Approach *
}

\author{
Frédéric Mazenc ${ }^{\dagger}$
}

\author{
Saeed Ahmed ${ }^{\ddagger}$
}

\author{
Hitay Özbay ${ }^{\ddagger}$
}

\begin{abstract}
We present a new trajectory based approach for state feedback stabilization of switched linear continuous-time systems with a time-varying input delay. In contrast with finding classical common Lyapunov function or multiple Lyapunov functions for establishing the stability of the closed-loop switched system, the new trajectory based approach relies on verifying certain inequalities along the solution of a supplementary system. This study does not make any assumption regarding the stabilizability of all of the constituent subsystems of the switched system. Moreover, no assumption is needed about the differentiability of the delay and no constraint is imposed on the upper bound of the delay derivative. Finally, an illustrative example is included to illustrate the applicability of our results.
\end{abstract}

Index Terms - Switched systems, delay, state feedback, stability.

\section{INTRODUCTION}

A switched linear continuous-time system is described by a finite set of Linear Time-Invariant (LTI) continuous-time subsystems (modes) and a switching signal (piecewise constant) which decides the mode to be activated at each instant of time. These systems have many practical applications; see [2], [3], [16], and [17].

There are three fundamental problems pertaining to the stability of switched systems, [10]; (i) finding conditions so that a switched system is stable for any arbitrary switching signal, (ii) determining important classes of switching signals for which a switched system is stable, and (iii) designing a switching signal that renders a switched system stable. In what follows, we review some classical solutions that have been reported in the literature for problem (i) and (ii). The most typical solution to problem (i) is to find conditions such that there exists a common quadratic Lyapunov function. A common quadratic Lyapunov function is a single quadratic Lyapunov function which is a global Lyapunov function for all of the modes of the switched system. A well known solution to problem (ii) in the literature is slowly switching signals i.e. if each subsystem is stable then the overall switched system is stable provided that the difference between consecutive switching time instants (which is termed as dwell-time) is sufficiently

\footnotetext{
*This work is supported by the PHC Bosphore 2016 France-Turkey under project numbers 35634QM (France) and EEEAG-115E820 (TÜBITTAK- The scientific and technological research council of Turkey).

${ }^{\dagger}$ Frédéric Mazenc is with EPI DISCO INRIA-Saclay, the Laboratoire des Signaux et Systèmes (L2S, UMR CNRS 8506), CNRS, CentraleSupélec, Université Paris-Sud, 3 rue Joliot Curie, 91192, Gif-sur-Yvette, France. frederic.mazenc@l2s.centralesupelec.fr

¥Saeed Ahmed and Hitay Özbay are with Department of Electrical and Electronics Engineering, Bilkent University, Ankara 06800, Turkey. \{ahmed, hitay\}eee.bilkent.edu.tr
}

large. This result can be established by using multiple Lyapunov functions. However, both common quadratic Lyapunov function based approach and dwell-time based results require to assume that all the modes of the switched system are stabilizable, [10]. This motivates us to study the stabilization of switched systems using dwell-time based results without requiring an assumption that all the constituent modes of the switched system are stabilizable. This idea is based on a new way to approach problem (ii). Moreover, motivated by the engineering applications of switched systems with time-delays in networked control systems [9], [11], and power systems [6], [15], we consider in this paper a time-varying delay in the input of the switched system.

To the authors' knowledge, very few contributions pertaining to the study of stabilization problem for switched systems with time-delays have been reported in literature up to now; see [5], [7], [8], [12], and [18] through [25]. In most of the contributions, the stability of the closed-loop switched systems was established by finding a common quadratic Lyapunov function or multiple Lyapunov functions. However, it has been shown in [4] that finding a common quadratic Lyapunov function even for a switched system with LTI modes is an NP-hard problem. Moreover, it becomes even more difficult to use classical Lyapunov based stability analysis methods for the case when a time-varying delay is present in the input of a switched system. Therefore, we propose a new and easier trajectory based approach to establish a robustness result with respect to presence of a pointwise delay in the input of a switched system. The trajectory based approach relies on verifying that there exist constants $v \in(0,1)$ and $\eta>0$ such that every trajectory $\kappa$ of a system satisfies a certain inequality of the form $|\kappa(t)| \leq v \sup _{l \in[t-\eta, t]}|\kappa(l)|$; see [13] and [14]. This method applies to a wide range of systems with timevarying delays. Moreover, we do not make any assumption about the stabilizability of all of the constituent subsystems of the switched system and impose no constraint on the upperbound of the derivative of the delay. In fact, we do not even assume that the delay is differentiable.

The rest of the paper is organized as follows. In Section II, some notations and preliminaries that will be utilized in Section III are introduced. Section III presents our main result based on a new trajectory based approach. Section IV provides an illustrating numerical example to demonstrate the effectiveness of our result. Finally, we summarize our findings and suggest some future research directions in Section V. 


\section{Notation AND PRELIMINARIES}

The notation will be simplified whenever no confusion can arise from the context. In what follows, all dimensions are arbitrary. The usual Euclidean norm of vectors, and the induced norm of matrices, are denoted by $|\cdot|$. Let $C^{1}$ denote the set of all continuously differentiable functions, where the domains and ranges will be clear from the context. Given any constant $\tau>0$, we let $C\left([-\tau, 0], \mathbb{R}^{n}\right)$ denote the set of all continuous $\mathbb{R}^{n}$-valued functions that are defined on $[-\tau, 0]$. We abbreviate this set as $C_{\text {in }}$, and call it the set of all initial functions. Also, for any continuous function $\varphi:[-\tau, \infty) \rightarrow \mathbb{R}^{n}$ and all $t \geq 0$, we define $\varphi_{t}$ by $\varphi_{t}(q)=\varphi(t+q)$ for all $q \in[-\tau, 0]$, i.e., $\varphi_{t} \in C_{\text {in }}$ is the translation operator. For each $\alpha \in \mathbb{N}$ and each function $\mathcal{F}:[0, \infty) \rightarrow \mathbb{R}^{\alpha}$, we set $\mathcal{F}\left(t^{-}\right)=\lim _{\beta \rightarrow t, \beta<t} \mathcal{F}(\beta)$ for all $t>0$.

Consider the switched linear continuous-time system described by

$$
\dot{x}(t)=A_{\sigma(t)} x(t)+B_{\sigma(t)} u(t)
$$

where $x \in \mathbb{R}^{n}$ is the state and $u \in \mathbb{R}^{p}$ is the control input. We consider a sequence $t_{k}$ such that there is a constant $\delta>0$ such that $t_{0}=0$ and $t_{k+1}-t_{k}=\delta$. Let $\sigma:[0, \infty) \rightarrow \mathcal{N}=$ $\{1, \ldots, N\}$ be a switching sequence such that for all $k \in \mathbb{N}$, there is $i \in \mathcal{N}$ such that $\sigma(t)=i$ for all $t \in\left[t_{k}, t_{k+1}\right) . A_{i}$ and $B_{i}$, for $i \in \mathcal{N}$, are state and input matrices which are assumed to be real and constant. In this paper, we consider the control input $u(t)$ in the following state-feedback form

$$
u(t)=K_{\sigma(t)} x(t-\tau(t))
$$

where the input delay $\tau$ is assumed to be a piecewise continuous function such that, for all $t \geq 0, \tau(t) \in[0, \bar{\tau}]$ with $\bar{\tau}>0$.

We introduce an assumption, which pertains to the stabilizability of the system (1), but does not imply that all the pairs $\left(A_{i}, B_{i}\right)$ are stabilizable.

Assumption 1. There are matrices $K_{i}$ and a switching law $\sigma$ such that the system

$$
\dot{\xi}(t)=\Omega_{\sigma(t)} \xi(t)+\varsigma(t)
$$

with

$$
\Omega_{i}=A_{i}+B_{i} K_{i}
$$

where $\varsigma$ is a piecewise continuous function, satisfies the following property: there are constants $T>\bar{\tau}, a \in[0,1)$, and $b \geq 0$ such that for all $t \geq T$

$$
|\xi(t)|^{2} \leq a|\xi(t-T)|^{2}+b \sup _{\ell \in[t-T, t]}|\varsigma(\ell)|^{2} .
$$

Remark 1. For more details on how to determine the constants $a, b, T$, and $\delta$ to satisfy Assumption 1 , the reader is refered to the journal version, [1].

\section{Main Results}

We are ready to state and prove our main result.

Theorem 1. If Assumption 1 holds, then there is a constant $\bar{\tau}>0$ such that if $\tau(t) \leq \bar{\tau}$ for all $t \geq 0$, then the control

$$
u(t)=K_{\sigma(t)} x(t-\tau(t))
$$

renders the origin of the system

$$
\dot{x}(t)=A_{\sigma(t)} x(t)+B_{\sigma(t)} u(t)
$$

Globally Uniformly Exponentially Stable (GUES).

Proof. Introducing the control input $u(t)=K_{\sigma(t)} x(t-\tau(t))$ into (2) yields the following closed-loop switched linear system:

$$
\dot{x}(t)=A_{\sigma(t)} x(t)+B_{\sigma(t)} K_{\sigma(t)} x(t-\tau(t))
$$

which can be modified as

$$
\dot{x}(t)=\left(A_{\sigma(t)}+B_{\sigma(t)} K_{\sigma(t)}\right) x(t)+B_{\sigma(t)} K_{\sigma(t)} z(t)
$$

with $z(t)=x(t-\tau(t))-x(t)$.

From the definition of $\Omega_{i}$, we obtain

$$
\dot{x}(t)=\Omega_{\sigma(t)} x(t)+B_{\sigma(t)} K_{\sigma(t)} z(t) .
$$

From Assumption 1, it follows that, for all $t \geq T+\bar{\tau}$,

$$
|x(t)|^{2} \leq a|x(t-T)|^{2}+b \sup _{\ell \in[t-T, t]}\left|B_{\sigma(\ell)} K_{\sigma(\ell)} z(\ell)\right|^{2} .
$$

Now, observe that

$$
\begin{aligned}
|z(t)| & \leq \int_{t-\bar{\tau}}^{t}|\dot{x}(m)| d m \\
& \leq \int_{t-\bar{\tau}}^{t}\left|\Omega_{\sigma(m)} x(m)+B_{\sigma(m)} K_{\sigma(m)} z(m)\right| d m \\
& \leq \bar{\tau} \sup _{\ell \in[t-\bar{\tau}, t]}\left|\Omega_{\sigma(\ell)} x(\ell)+B_{\sigma(\ell)} K_{\sigma(\ell)} z(\ell)\right| .
\end{aligned}
$$

It follows that

$$
\begin{aligned}
|z(t)|^{2} \leq & 2 \bar{\tau}^{2} \sup _{\ell \in[t-\bar{\tau}, t]}\left|\Omega_{\sigma(\ell)} x(\ell)\right|^{2} \\
& +2 \bar{\tau}^{2} \sup _{\ell \in[t-\bar{\tau}, t]}\left|B_{\sigma(\ell)} K_{\sigma(\ell)} z(\ell)\right|^{2} .
\end{aligned}
$$

Using the following constants:

$$
s_{1}:=\sup _{i \in \mathcal{N}}\left|B_{i} K_{i}\right|, \quad s_{2}:=\sup _{i \in \mathcal{N}}\left|\Omega_{i}\right|,
$$

we deduce from (4) and (5) that

$$
|x(t)|^{2} \leq a|x(t-T)|^{2}+b s_{1}{ }^{2} \sup _{\ell \in[t-T, t]}|z(\ell)|^{2}
$$

and

$$
\begin{aligned}
|z(t)|^{2} \leq & 2 s_{2}{ }^{2} \bar{\tau}^{2} \sup _{\ell \in[t-\bar{\tau}, t]}|x(\ell)|^{2} \\
& +2 s_{1}{ }^{2} \bar{\tau}^{2} \sup _{\ell \in[t-\bar{\tau}, t]}|z(\ell)|^{2} .
\end{aligned}
$$

To analyze the consequences of these inequalities, we introduce the function

$$
\Lambda(x(t), z(t))=|x(t)|^{2}+r|z(t)|^{2}
$$


where $r>0$ is a constant to be selected later. The inequalities (6) and (7) imply that, for all $t \geq T+\bar{\tau}$,

$$
\begin{aligned}
\Lambda(x(t), z(t)) \leq & a|x(t-T)|^{2}+b s_{1}^{2} \sup _{\ell \in[t-T, t]}|z(\ell)|^{2} \\
& +2 s_{2}^{2} r \bar{\tau}^{2} \sup _{\ell \in[t-\bar{\tau}, t]}|x(\ell)|^{2} \\
& +2 s_{1}^{2} r \bar{\tau}^{2} \sup _{\ell \in[t-\bar{\tau}, t]}|z(\ell)|^{2} \\
\leq & a \Lambda(x(t-T), z(t-T)) \\
& +\frac{b s_{1}^{2}}{r} \sup _{\ell \in[t-T, t]} \Lambda(x(t), z(t)) \\
& +2 s_{2}^{2} r \bar{\tau}^{2} \sup _{\ell \in[t-\bar{\tau}, t]} \Lambda(x(t), z(t)) \\
& +2 s_{1}^{2} \bar{\tau}^{2} \sup _{\ell \in[t-\bar{\tau}, t]} \Lambda(x(t), z(t)) .
\end{aligned}
$$

Consequently,

$$
\begin{aligned}
\Lambda(x(t), z(t)) \leq & \left(a+\frac{b s_{1}^{2}}{r}+2 s_{2}^{2} r \bar{\tau}^{2}+2 s_{1}^{2} \bar{\tau}^{2}\right) \\
& \times \sup _{\ell \in[t-T, t]} \Lambda(x(\ell), z(\ell)) .
\end{aligned}
$$

Choosing, for instance, $r=\frac{2 b s_{1}^{2}}{1-a}$, we obtain

$$
\begin{aligned}
\Lambda(x(t), z(t)) \leq & {\left[\frac{a+1}{2}+\left(\frac{4 b s_{2}^{2}}{1-a}+2\right) s_{1}^{2} \bar{\tau}^{2}\right] } \\
& \times \sup _{\ell \in[t-T, t]} \Lambda(x(\ell), z(\ell)) .
\end{aligned}
$$

Since $\frac{a+1}{2}<1$, we conclude that if $\bar{\tau}$ is such that

$$
\frac{a+1}{2}+\left(\frac{4 b s_{2}^{2}}{1-a}+2\right) s_{1}^{2} \bar{\tau}^{2}<1
$$

then the origin of (2) is GUES (see [13]), which gives the condition

$$
\bar{\tau}<\frac{1-a}{2 s_{1}} \sqrt{\frac{1}{2 b s_{2}^{2}+1-a}} .
$$

This concludes the proof.

\section{Numerical Example AND Simulation}

Consider the closed-loop switched linear time-delay system given by

$$
\dot{x}(t)=A_{\sigma(t)} x(t)+B_{\sigma(t)} K_{\sigma(t)} x(t-\tau(t))
$$

with $x \in \mathbb{R}^{2}, \tau \in[0, \bar{\tau}]$ for any $\bar{\tau}>0, \sigma:[0,+\infty) \rightarrow$ $\mathcal{N}=\{1,2\}$,

$$
A_{1}=\left[\begin{array}{ll}
0 & 0 \\
0 & \frac{1}{2}
\end{array}\right], \quad B_{1}=\left[\begin{array}{cc}
-\frac{4}{5} & 0 \\
0 & 0
\end{array}\right]
$$

and

$$
A_{2}=\left[\begin{array}{cc}
0 & \frac{1}{2} \\
-\frac{1}{2} & 0
\end{array}\right], B_{2}=\left[\begin{array}{cc}
-\frac{1}{3} & 0 \\
0 & 2
\end{array}\right] .
$$

It is easy to verify that the subsystem 1 is not stabilizable whereas the subsystem 2 is stabilizable.

We choose the linear state feedback gain $K_{i}$ corresponding to each subsystem of (9) as

$$
K_{1}=\left[\begin{array}{cc}
\frac{1}{2} & 0 \\
0 & 0
\end{array}\right], K_{2}=\left[\begin{array}{cc}
9 & 0 \\
0 & -\frac{3}{2}
\end{array}\right] .
$$

Then, the feedback subsystem $\Omega_{i}=A_{i}+B_{i} K_{i}$ is given by

$$
\Omega_{1}=\left[\begin{array}{cc}
-\frac{2}{5} & 0 \\
0 & \frac{1}{2}
\end{array}\right], \Omega_{2}=\left[\begin{array}{cc}
-3 & \frac{1}{2} \\
-\frac{1}{2} & -3
\end{array}\right] .
$$

The eigenvalues for the feedback subsystems $\Omega_{1}$ and $\Omega_{2}$ are $\left\{\frac{1}{2},-\frac{2}{5}\right\}$ and $\left\{-3 \pm j \frac{1}{2}\right\}$, respectively. Therefore, the matrix $\Omega_{1}=A_{1}+B_{1} K_{1}$ is not Hurwitz whereas the matrix $\Omega_{2}=A_{2}+B_{2} K_{2}$ is Hurwitz. The constants $s_{1}$ and $s_{2}$ can be computed as

$$
s_{1}=\sup _{i \in\{1,2\}}\left|B_{i} K_{i}\right|=3
$$

and

$$
s_{2}=\sup _{i \in\{1,2\}}\left|\Omega_{i}\right|=3.0414 .
$$

We choose $a=0.0143, b=6.9813, T=4$ which gives us $0<\bar{\tau}<0.0144$. Using the idea presented in [1], one can easily substantiate that (4) is satisfied with our choice of $a, b$, $T, K_{1}, K_{2}$, and a periodic switching signal $\sigma(t)$ with $\delta=1$ shown in Fig. 1d.

Fig. 1 shows the simulation of the feedback switched linear system (9) for a time-varying delay $\tau(t)$ of Fig. 1a with an initial condition $x_{o}=\left[\begin{array}{ll}-3 & 2\end{array}\right]^{T}$ using a periodic switching signal shown in Fig. 1d. The state trajectories and phase portrait depicted in Figs. $1 b$ and $1 \mathrm{c}$ verify the GUES of the closed-loop switched time-delay system (9).

Remark 2. The dwell-time based approach presented in [20] fails for our example because it requires to assume that all the constituent modes of the switched system are controllable. Moreover, our results are better suited to certain applications because we do not require to impose any constraint on the upper bound of the delay derivative as opposed to [18].

\section{CONCluding Remarks}

In this paper, we presented state feedback stabilization results for switched linear systems with a time-varying input delay. We adopted a new trajectory based approach instead of a Lyapunov method. This made it possible to obtain results that are not based on restrictive assumptions that are traditionally made in the literature regarding the stability and design of switched systems with delay such as stabilizability of all of the constituent subsystems, differentiability of the delay or upper bound on the delay derivative. Some open problems to be addressed in future studies include: (i) extending these results to the output feedback case, (ii) exhibiting a systematic way to determine the constants $a, b$ and $T$ to satisfy Assumption 1 for computing an explicit value of $\bar{\tau}$ for some broad families of systems, and (iii) achieving less conservative results for $\bar{\tau}$ to guarantee the GUES of the closed-loop switched time-delay system.

\section{REFERENCES}

[1] S. Ahmed, F. Mazenc, and H. Özbay. Dynamic output feedback stabilization of switched linear systems with delay via a trajectory based approach. Submitted for publication, 2017. 
[2] S. Ahmed and H. Özbay. Switching robust controllers for automatic regulation of postoperative hypertension using vasodilator drug infusion rate. In Proceedings of IFAC Workshop on Linear Parameter Varying Systems, Grenoble, France, 2015, pp. 224-229.

[3] S. Ahmed and H. Özbay. Design of a switched robust control scheme for drug delivery in blood pressure regulation. In Proceedings of IFAC Workshop on Time Delay Systems, Istanbul, Turkey, 2016, pp. 252-257.

[4] V. Blondel and J. N. Tsitsiklis. NP-hardness of some linear control design problems. SIAM Journal on Control and Optimization 35(6):21182127, 1997.

[5] S. Y. Calıskan, H. Özbay, and S.-I. Niculescu. Dwell-time computation for stability of switched systems with time delays. IET Control Theory \& Applications 7(10):1422-1428, 2013.

[6] F. F. Digham and M .S. Alouini. Variable-rate noncoherent M-FSK modulation for power limited systems over Nakagami-fading channels. IEEE Transactions on Wireless Communications 3(4):1295-1304, 2004.

[7] L. Hetel, J. Daafouz, and C. Iung. Stabilization of arbitrary switched linear systems with unknown time-varying delays. IEEE Transactions on Automatic Control 51(10):1668-1674, 2006.

[8] S. Kim, S. A. Campbell, and X. Liu. Stability of a class of linear switching systems with time delay. IEEE Transactions on Circuits and Systems 53(2):384-393, 2006

[9] D. K. Kim, P. Park, and J. W. Ko. Output-feedback $\mathcal{H}_{\infty}$ control of systems over communication networks using a deterministic switching system approach. Automatica 40(7):1205-1212, 2004.

[10] D. Liberzon and A. S. Morse. Basic problems in stability and design of switched systems. IEEE Control Systems Magazine 19(5):59-70, 1999.

[11] G. P. Liu, Y. Xia, D. Rees, and W. S. Hu. Design and stability criteria of networked predictive control systems with random network delay in the feedback channel. IEEE Transactions on Systems, Man, and Cybernetics 37(2):173-184, 2007.

[12] M. S. Mahmoud. Switched Time-Delay Systems: Stability and Control. Springer, New York, USA, 2010.

[13] F. Mazenc and M. Malisoff. Trajectory based approach for the stability analysis of nonlinear systems with time delays. IEEE Transactions on Automatic Control 60(6):1716-1721, 2015.

[14] F. Mazenc, M. Malisoff, and S.-I. Niculescu. Stability analysis for systems with time-varying delay: trajectory based approach. In Proceedings of IEEE Conference on Decision and Control, Osaka, Japan, 2015, pp. 1811-1816.

[15] C. Meyer, S. Schröder, and R. W. De Doncker. Solid-state circuit breakers and current limiters for medium-voltage systems having distributed power systems. IEEE Transactions on Power Electronics 19(5):13331340, 2004

[16] A. S. Morse. Control Using Logic-Based Switching. Springer-Verlag, London, UK, 1997.

[17] Z. Sun and S. S. Ge. Switched Linear Systems: Control and Design. Springer, London, UK, 2005.

[18] X.-M. Sun, W. Wang, G. -P. Liu, and J.Zhao. Stability analysis for linear switched systems with time-varying delay. IEEE Transactions on Systems, Man, and Cybernetics-Part B: Cybernatics 38(2):528-533, 2008.

[19] X.-M. Sun, J. Zhao, and D. J. Hill. Stability and $\mathcal{L}_{2}$ gain analysis for switched delay systems: A delay-dependent method. Automatica 42(10):1769-1774, 2006.

[20] L. Vu and K. A. Morgansen. Stability of time-delay feedback switched systems. IEEE Transactions on Automatic Control 55(10):2385-2390, 2010.

[21] M. Xiang, Z. Xiang, and H. R. Karimi. Stabilization of positive switched systems with time-varying delays under asynchronous switching. International Journal of Control, Automation and Systems 12(5):939-947, 2014.

[22] G. Xie and L. Wang. Stability and stabilization of switched linear systems with state delay. In Proceedings of Mathematical Theory of Networks and Systems Conference, Leuven, Belgium, 2004.

[23] G. Xie and L. Wang. Stabilization of switched linear systems with timedelay in detection of switching signal. Journal of Mathematical Analysis and Applications 305(1):277-290, 2005.

[24] P. Yan and H. Özbay. Stability analysis of switched time delay systems. SIAM Journal on Control and Optimization 47(2):936-949, 2008.

[25] P. Yan, H. Özbay, and M. Sansal. Robust stabilization of parameter varying time delay systems by switched controllers. Applied and Computational Mathematics 13(1):31-45, 2014

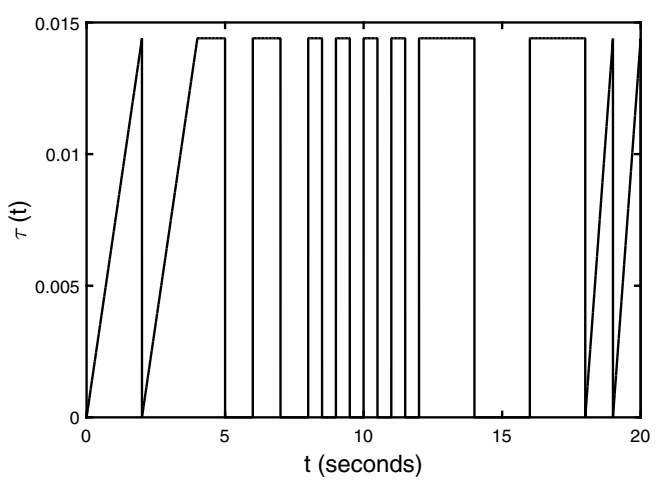

(a) Time varying delay $\tau(t)$

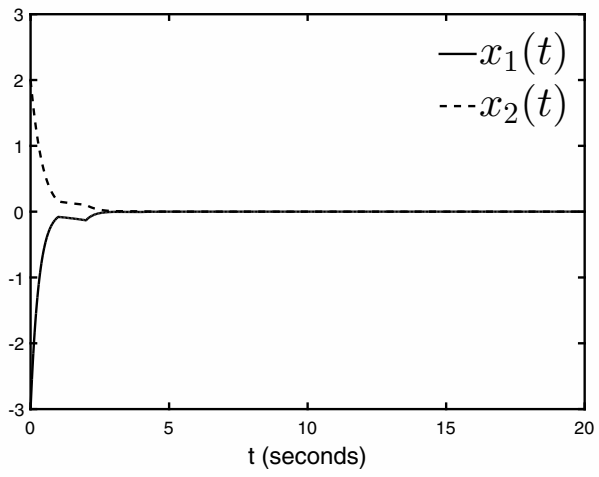

(b) Trajectories of state $x$

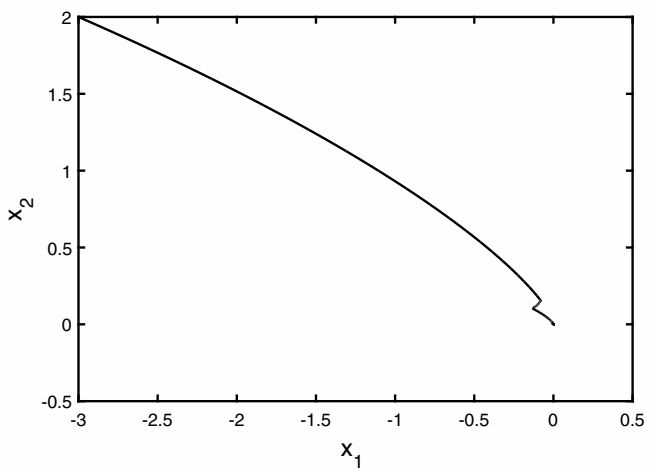

(c) Phase Portrait

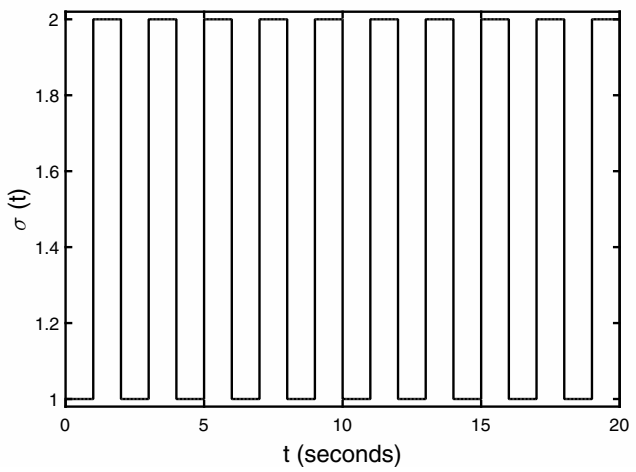

(d) Switching Signal $\sigma(t)$

Fig. 1: Illustration of Theorem 1 and simulation of (9). 